\title{
ON THE HYDROGEN ABUNDANCE IN WOLF-RAYET STARS
}

\author{
Werner Schmutz \\ Institute of Astronomy \\ li'T-Zentrum CH-8092 Zürich \\ Switzerland
}

\section{ABSTRAC'T}

With a semiempirical NLTE-model in spherical geometry we investigate the hydrogen abundance in Wolf-Rayct stars. We find that the flux contribution of the H-Balmer to the Ife-Pickering lines lies between the usually applied approximate formulae for the optically thin and optically thick case. This result can be understood with our finding that there are no optically thin helium and hydrogen lines. The influence of the temperature structure on the relative intensity of the hydrogen-helium blends to the helium lines is vanishingly small, even for peculiar temperature laws. 'The conclusion is that Wolf-Rayet stars are hydrogen deficient objects.

\section{INTIRODUCTION}

The majority of Wolf-Rayet specialists are convinced that the elemental abundances in Wolf-lRayel stars are abnormal, especially that they are hydrogen deficient. However, up to now there have been no elaborate investigations of the formation of the helium and hydrogen lines in the extended atmospheres of Wolf-Rayet stars. To analyse the Balmer and Pickering lines the approximale formula of Castor and van Blerkom (1970, henreforth (,B) for the optically thin case has usually been applied

$$
\Delta \log \left(W_{\lambda}\right) \approx \log \left(\begin{array}{c}
N\left[\mathrm{H}^{+}\right] \\
N\left[\mathrm{He}^{++}\right]
\end{array}\right) \approx \log \left(\begin{array}{c}
N[\mathrm{H}] \\
N[\mathrm{H} \mathrm{c}]
\end{array}\right),
$$

where $\Delta \log \left(W_{\lambda}\right)=\log (I(\mathrm{He}+\mathrm{H}) / I(\mathrm{He}))$ is the ratio of the intensity of a Balmer-l'ickering blend to the estimated intensity of the Pickering line alone. The optically thin approximation has been justified by CB, who showed that the Pickering lines from the upper level $n>10$ are optically thin. Hlowever, the model used by CB is a one point model. This was criticised e.g. by Underhill (1982) or by Sahade (1981), who writes: "(...) at least we ought to be cautious and not state so firmly that Wh stars are ll-deficent objects." The approximation of Massey (1980) for the optically thick case takes into account the different emitting volumes of the lines. But the lines used to derive the hydrogen abundance are not expected to be optically thick.

The purpose of this paper is to investigate the formation of III and Ifell lines in a spherically extended atmosphere and the influence of the temperature structure on these line intensities. 


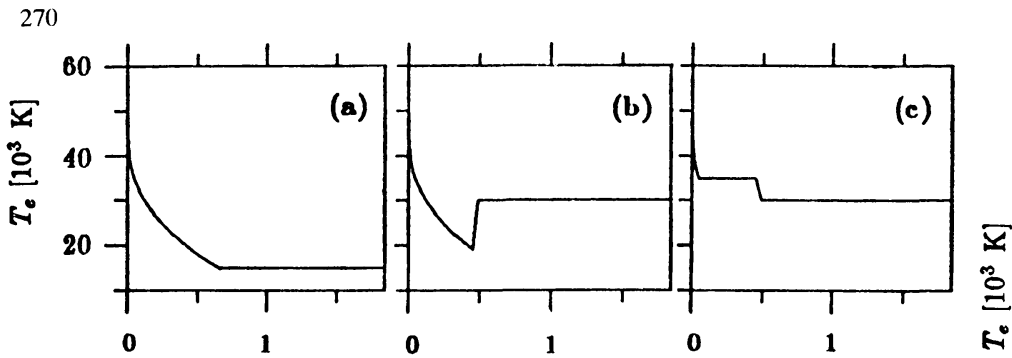

Figure 1. The temperature laws used for the model calculations: (a) the law used in section $3 a,(b)-(d)$ the laws used in section $3 \mathrm{~b}$. The continuum optical depth $r_{R o e r}=1 / 3$ is at $\log \left(r / R_{c}\right) \approx 0.25$.

\section{THE MODEL ATMOSPHERE}

W. SCHMUTZ

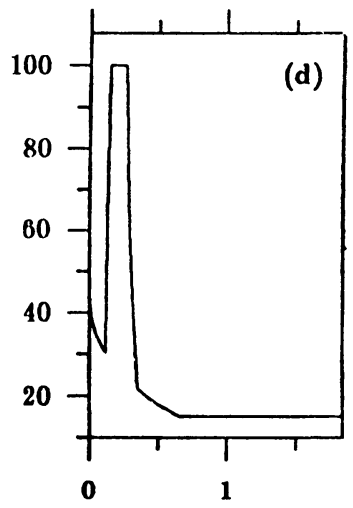

$\log \left(r / R_{c}\right)$

The model is semiempirical, i.e. the velocity law $V(r)$ and the temperature law $T(r)$ are free input parameters. Further physically important input parameters are the mass loss rate and the abundances. The elements included in the calculation are hydrogen and helium. The calculation of the continuum radiation field and the level populations are performed alternately until convergence of the level populations (' $\Lambda$-iteration'). The NLTE level populations are calculated with the Sobolev approximation of Castor (1970), modified to treat the diffuse radiation field. Interaction between the helium and hydrogen lines of the same wavelength are taken into account by using the same combined escape probability for both elements. We set the inner boundary $R_{c}$ where the velocity field is $V\left(R_{c}\right)=$ $V_{(x)} / 100$; we do not include deeper layers because the validity of the Sobolev approximation would break down. At the inner boundary we assume the outgoing radiation field to be $I^{o u t}\left(\nu, R_{c}\right)=B\left(\nu, T\left(R_{c}\right)\right)$ and LTE level population. For the models presented here the inner boundary lies al $\tau_{R O \theta} \approx 8$, therefore the observable quantities are not very sensitive to the boundary conditions.

Following Klein and Castor (1978) we write for the equivalent widths

$W_{\lambda}=\sum_{L_{l,},}^{h \nu} \int 4 \pi r^{2}\left[\beta^{o u t} N_{u} A_{u, l}-\left(N_{l} B_{l, u}-N_{u} H_{u, l}\right) J^{o u t}\right] e^{-r_{\nu}^{\prime}(r)} d r=\int E(r)+\Lambda(r) d r$,

where $\beta^{\text {out }}$ is the escape probability of a line photon to leave the atmosphere and $J^{\text {out }}$ is the mean outgoing conlinuum radiation seen by the line al the position $r$.

For this investigation only the abundances and the temperature law (given in figure 1) are varied. The assumed mass loss rate is $\delta M / \partial t=4 \cdot 10^{-5} M_{(} /$year and the velocity law is

$$
V(r)=3000\left(1-\frac{R_{c}}{r}\right)^{1}[\mathrm{~km} / \mathrm{sec}]
$$

with $R_{c}=5.5 R_{(0)}$. Though we have no particular star in mind we notice that the resulting flux and line strenglhs resemble the LMC WN4 star FD13 (Smith and Willis, 1983).

Table 1.

Intensity ratio $\Delta \log \left(W_{\lambda}\right)=$ $\log (I(\mathrm{He}+\mathrm{H}) / I(\mathrm{He}))$ of the l'ickering-Balmer blends to the Pickering line intensity.

$\left[\begin{array}{c|c}N[\mathrm{H}] / N[\mathrm{He}] & \text { opt. thin } \\ \hline 2.0 & 0.47 \\ \hline 1.0 & 0.30 \\ 0.5 & 0.18 \\ 0.1 & 0.040\end{array}\right.$

\begin{tabular}{|c|c|}
\hline opt. thick & Model \\
\hline 0.32 & 0.41 \\
0.20 & 0.24 \\
0.12 & 0.15 \\
0.027 & 0.030 \\
\hline
\end{tabular}




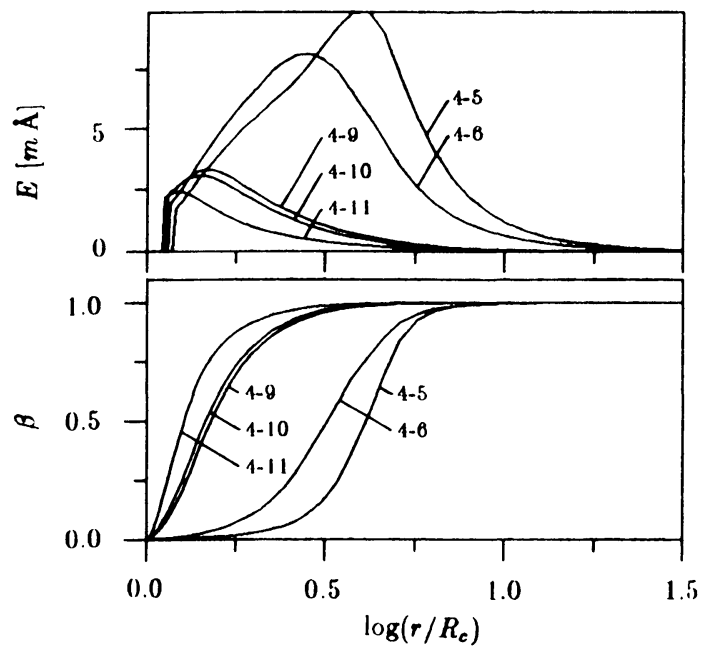

Figure 2a. He II and combined $\mathrm{H}$ I and He II line emission $E(r)$ (sce text) as a function of radius for a model with $N[\mathrm{H}] / N[\mathrm{He}]$ $=1$. The labels on the curves refer to the He II transitions. The curves 4-6 and 1-10 include the contribution from II I 2-3 respectively $2-5$.

ligure $2 b$. The escape probability $\beta(r)$ as a function of radius for the same transitions as in figure $2 a$.

\section{RESULTS OF THE MODEL CALCULATIONS}

(a) The Balmer-Pickering line strengths for different abundances

In Table 1 we give the calculated contribution of the Balmer to the Pickering lines for four different hydrogen abundances. Also given are the corresponding values of the two approximate formulae. It is interesting to note that our value lies between the two approximations. To understand this result we present figure $2 a$, where the quantily $E(r)$ from the formula above is plotted as a function of radius. In figure $2 b$ we give the escape probability $\beta(r)$. A comparison of these two graphs shows that the main contribution to the line strength arises from the region where $\beta \approx 0.5$. This is true for all lines (except for optically thick ones), even for those from higher $n$. Hence, there are no optically thin He lines; the result of ClB was an effect of the one point approximation.

The figures of Conti et al. (1983) give an idea of the quality of the observations: the detection limit is $N\left[\mathrm{H}^{+}\right] / N\left[\mathrm{He}^{++}\right] \approx 0.3$.

(b) The influence of the temperature structure

For the calculations reported in section 3 a the temperature law of figure 1 a was used. To analyse the influence of the temperature law, we ran the three different temperature structures given in figures $1 \mathrm{~b}, 1 \mathrm{c}$ and $1 \mathrm{~d}$. Though the absolute line strengths are dependent on the laws we do not find any measurable change of $\Delta \log W_{\lambda}$. This is due to $\mathrm{H}^{+}$and $\mathrm{He}^{++}$being isoelectronic. If both hydrogen and helium are fully ionized, line emissivities of $\mathrm{H}$ I and He Il react practically in the same way to variations in $N_{e}$ and $T_{e}$. This implies also that an inhomogenious atmosphere, as suggested by Underhill (1983), would have no influence on the relative line strengths of hydrogen to helium.

\section{CONCLUSION}

We found that there is no mechanism to suppress the $\mathrm{H} \mathrm{I} / \mathrm{He} \Pi \mathrm{I}$ line ratio. Neither optical thickness, i.e. density structure, nor a peculiar temperature law changes the relative emission. An upper and a lower limit on the $N\left[\mathrm{H}^{+}\right] / N\left[\mathrm{He}^{++}\right]$ratio can be set by the approximate formulae. As under all conditions $\mathrm{He}{ }^{++}$will recombine before $\mathrm{II}^{+}$, the Pickering and Balmer line intensities will give an upper limit for the hydrogen abundances. 


\section{ACKNOWLEDGEMENTS}

This research has been supported by the Swiss Science Foundation.

\section{REFERENCES}

Castor,J.I.: 1970, Monthly Notices Roy. Astron. Soc. 149,111.

Castor,J.I. und van Blerkom,D.: 1970, Astrophys. J. 161,485.

Conti,P.S.,Lenp,E.M. und Perry,D.N.: 1983 Astrophys. J. 268,228.

Klein,R.I. und Castor,J.J.: 1978, Astrophys. J. 220,902.

Massey,P.: 1980, Astrophys. J. 236,526.

Sahade,J.: 1981, Rev. Mexicana Astron. Astrof. 6,189.

Smith,L..J. und Willis, ^.J.: 1983, Astron. Astrophys. suppl., in press.

Underhill,A.B.: 1982, 'Wolf-Rayet Stars: Observations, Physics, Evolution', W.H.de Loore und $\Lambda$.J.Willis (eds.), IAU Symp. 99,p19.

Under hill,A.I3.: 1983, Astrophys. J. $265,833$.

\section{DISCUSSION}

Kudritzki: Did you include the HeII-resonance lines in your rate equations and did you encounter then convergence difficulties within the $\Lambda$-iteration scheme?

Schmutz: Yes, I include the HeII-resonance lines and I got indeed problems namely that the convergence is very slow. On the basis of a few tests

I think that it is a real convergence. 